Sains Malaysiana 51(1)(2022): 27-38

http://doi.org/10.17576/jsm-2022-5101-03

\title{
Super- or Single Infection: Wolbachia Supergrouping of Wild Mosquito Populations from Varied Location Types in Peninsular Malaysia
}

(Jangkitan Super atau Tunggal: Superkumpulan Wolbachia Populasi Nyamuk Liar dari Pelbagai Jenis Lokasi di Semenanjung Malaysia)

\author{
Noor Shazleen Husnie Mohd Mohtar, Emelia Osman, Mohd Farihan Md Yatim \& Aishah Hani AzIL*
}

\section{ABSTRACT}

Wolbachia has the ability to cause reproductive abnormalities in infected hosts including cytoplasmic incompatibility (CI). CI is activated when there are multiple Wolbachia supergroups or strains infection present in insect populations. Wolbachia-transinfected mosquitoes have been used widely in some countries as a biological control agent. In order to ensure a successful Wolbachia establishment, it is important to determine the diversity of natural Wolbachia present in the wild mosquito populations. The adults and immature stages of mosquitoes were collected from urban, suburban and rural areas and were reared into adults and identified to species before being subjected to molecular analysis. We found that $22 \%$ out of 222 males and $34.6 \%$ of 543 females tested were carrying Wolbachia based on PCR amplification of the Wolbachia 16S rDNA genes technique. PCR digestion for Wolbachia supergrouping showed that most of the Ae. albopictus were superinfected with Wolbachia (52.41\%), whereas $21 \%$ and $28 \%$ of the positive samples were singly infected with supergroup $A$ and $B$, respectively. There is an indication that prevalence of Wolbachia varies between mosquito populations in different areas. However, further studies to incorporate both PCR amplication of the Wolbachia 16S rDNA and wsp genes with bigger sample size should be performed to measure exact infection of Wolbachia in Malaysia. The baseline data on diversity of Wolbachia supergroups is expected to facilitate Wolbachia strategy by helping us to better understand the patterns and impact of the bacteria's transmission in the environment.

Keywords: $16 S$ rDNA; Culicidae; PCR digestion; Wolbachia supergroup

\section{ABSTRAK}

Wolbachia berkebolehan menyebabkan keabnormalan reproduktif kepada perumah yang dijangkitinya, antaranya ketidakserasian sitoplasma (CI). CI diaktifkan apabila terdapat kepelbagaian jangkitan daripada superkumpulan atau strain Wolbachia yang hadir di dalam sesuatu populasi. Nyamuk transjangkitan Wolbachia ini telah digunakan secara meluas di sesetengah negara sebagai agen kawalan biologi. Namun bagi memastikan keberjayaan Wolbachia untuk bermandiri, adalah penting untuk mengenal pasti kepelbagaian Wolbachia yang hadir secara semula jadi di dalam populasi nyamuk liar. Nyamuk peringkat dewasa dan pra-matang disampel daripada kawasan bandar, pinggir bandar dan pedalaman yang kemudiannya dibiak sehingga dewasa dan spesiesnya dikenal pasti sebelum diteruskan dengan analisis molekul. Berdasarkan kaedah amplifikasi PCR yang menyasarkan gen 16S rDNA, kajian mendapati 22\% daripada 222 nyamuk jantan dan 34.6\% daripada 543 betina membawa Wolbachia. Pencernaan produk PCR dilakukan bagi menentukan super-kumpulan Wolbachia dan hasilnya majoriti Aedes albopictus dijangkiti Wolbachia daripada kedua-dua superkumpulan A dan B (52.41\%) manakala 21\% dan 28\% daripadanya masing-masing terjangkit secara tunggal, superkumpulan A dan B. Ini menandakan taburan kumpulan Wolbachia adalah berbeza antara populasi nyamuk di kawasan yang berbeza. Namun, kajian lanjutan yang melibatkan sampel saiz yang lebih besar serta gabungan penggunaan dua gen Wolbachia $16 \mathrm{~S}$ rDNA dan wsp amat diperlukan bagi mengukur kadar jangkitan Wolbachia di Malaysia. Data garis dasar mengenai kepelbagaian superkumpulan Wolbachia yang hadir dijangka dapat membantu mempermudahkan untuk memahami taburannya dan kesan penyebarannya pada persekitaran.

Kata kunci: 16 S rDNA gen; Culicidae; pencernaan PCR; superkumpulan Wolbachia 


\section{INTRODUCTION}

Outbreaks of dengue and chikungunya are occurring in Malaysia for the past few years with a higher number of dengue cases reported in $2019(130,101)$ compared to $2018(80,615)$. However, the number of dengue cases reported until 7 November 2020 was 83,752, indicating a reduction of $25.5 \%$ compared to previous year (KKM 2020, 2019). These diseases are transmitted by Aedes aegypti and Aedes albopictus. Previously, Ae. albopictus is predicted to be the main vector responsible for transmission of DENV during the dengue outbreak in China mainland (Luo et al. 2017; Xu et al. 2007). Meanwhile, in Malaysia, Ae. albopictus was abundantly found in urban residential areas, for example Kampung Baru which are located at the centre of Kuala Lumpur (Chen et al. 2006; Rozilawati et al. 2015). Thus, this species could serve as a potential vector for the virus transmission in the areas. Due to increasing reported cases of the vector-borne diseases, extensive control must be planned and executed including those that target the vector. However, high usage of thermal spraying or fogging can result in the development of insecticide resistance in the vectors (Hamdan et al. 2005; Loke et al. 2010). Therefore, biological control which involves the release of Wolbachia-transinfected Ae. aegypti are suggested to be added to complement existing vector control methods. This method is predicted to limit the transmission of dengue viruses by manipulating the Aedes populations.

Releases of females or males infected with selected strain of Wolbachia will play a major role in the successful of population replacement and suppression. The first release of Ae. aegypti transinfected Wolbachia (w Mel strain) were conducted in Cairns region of northern Queensland, Australia and a successful of Wolbachia establishment in the released mosquito populations has been reported (Hoffman et al. 2011). Such releases were conducted via the World Mosquito Program involving several dengue-affected countries including Fiji which has undertaken $w \mathrm{Mel}$ mosquito deployment in 2018 (WMP 2019). Meanwhile, the Wolbachia project in Malaysia involves the releases of Ae. aegypti carrying Wolbachia strain of $w \mathrm{AlbB}$ at six localities of dengue endemic areas (Nazni et al. 2019). Two mechanisms are predicted to occur. First mechanism is population replacement which takes place when infected females mate with uninfected males or infected males carrying same supergroup or strain in the field which will result in all progeny carrying Wolbachia. Second, population suppression which occurs when males infected with
Wolbachia cross-mate with infected females carrying different strain or uninfected females in the field which will produce unhatched eggs. The successful establishments of introduced Wolbachia strains were recorded in Australia and Malaysia (Hoffmann et al. 2011; Nazni et al. 2019).

However, the releases of Wolbachia-infected mosquitoes have been involving only Ae. aegypti thus far. It is more straightforward to introduce transinfected Ae. aegypti into the aposymbiotic mosquito populations compared to Ae. albopictus populations, which naturally carry Wolbachia. Many surveys have been conducted on the prevalence of natural Wolbachia strain present in various mosquito species and most of the Ae. albopictus samples collected were found to be positive with Wolbachia (Kittayapong et al. 2000; Nugapola et al. 2017; Rasgon \& Scott 2004; Ricci et al. 2002; Zhou et al. 1998). Wolbachia does not induce pathogen interference when it naturally lives inside the host (Mousson et al. 2012). Wolbachia act as a potential gene-driving system by manipulating vector populations when they are artificially infected and cause a wide range of reproductive abnormalities called cytoplasmic incompatibility (CI) (Sinkins 2004). The CI activation enables Wolbachia to spread rapidly and replace the uninfected mosquito populations with introduced strain. Several studies have shown the successful development of Wolbachia-transinfected Ae. albopictus through the embryonic microinjection with the selected strains and the capability to suppress the population of Ae. albopictus was demonstrated in the laboratory (Calvitti et al. 2015; Fu et al. 2010; Zhang et al. 2016). The successful development of these strains is useful for future dengue vector control by targeting endemic areas which are abundant with Ae. albopictus populations.

However, a better understanding on natural Wolbachia infection in the populations are needed before Wolbachia-transinfected Ae. albopictus are released. Therefore, our main objective was to determine the distribution of Wolbachia supergroups infection in the selected mosquito populations located in Peninsular Malaysia using a conventional method, PCR targeting $16 \mathrm{Sr}$ DNA gene and supergrouping by PCR-digestion. Based on study conducted by Marcon et al. (2011), both target gene $16 \mathrm{~S}$ rDNA and wsp have been proved to be highly specific and sensitive in detection of Wolbachia. The primers designed targeting $16 \mathrm{~S}$ rDNA have been proved as stand-alone primers which can be used both as detection and Wolbachia supergroup classification (Marcon et al. 2011) but wsp gene is more preferable 
for the phylogenetic study. Furthermore, this study contributes to the current knowledge about Wolbachia strains prevalence in wild mosquito populations of a variety of species and locations.

\section{MATERIALS AND METHODS}

\section{STUDY AREA AND MOSQUITO COLLECTIONS}

This cross-sectional study was conducted in randomly selected areas involving several states in Peninsular Malaysia. Mosquitoes were sampled from March 2014 to May 2015 in several localities (Table 1), before the Wolbachia releases project by Ministry of Health which were conducted in another localities. Selected localities were classified as urban and rural areas. Mosquitoes from various species were collected using several collection methods; BG-Sentinel trap and human landing catch (HLC) for adults. Immature stages were collected using mosquito larvae trapping devices (MLTD) and larva survey. Most of the samplings were conducted during one-site-visit of each location such as BG-Sentinel, HLC and larva survey. MLTD or also known as autocidal trap have been used by Vector Unit of Kuala Lumpur City Hall (DBKL) as surveillance tools for dengue vector. MLTD is made from a cylindrical shape plastic container $(24$ x 13.5 $\mathrm{cm}$ ), black funnel, cap and a jacket (black polybag) which used to cover the transparent container (Zainol-Ariffin et al. 2009). The MLTD was filled with approximately $1 \mathrm{~L}$ of dechlorinated water and monitored every week for the presence of larvae and eggs. All of these methods were used to maximize collections for both immature and adult stages from each location. The collected larvae and pupae were brought to the insectarium and reared into adults. Adult mosquitoes were identified using several keys of identification and were sorted according to species. Mosquitoes were kept in $95 \%$ ethanol and stored at -20 ${ }^{\circ} \mathrm{C}$ prior to DNA extraction.

\section{Wolbachia DETECTION}

Genomic DNA extraction procedure was conducted according to manufacturer's protocol with several modifications as stated by Noor-Shazleen-Husnie et al. (2018). The DNA extractions were performed by homogenizing whole body of adult mosquito, individually in $100 \mu \mathrm{L}$ of DNAzol ${ }^{\circledR}$ reagents (Life Technologies, USA). Subsequently, $50 \mu \mathrm{L}$ of absolute ethanol AR (Ajax Finechem Pty. Ltd., Australia) were added to the supernatant to precipitate the DNA. The DNA was washed twice with $75 \%$ ethanol before been eluted with $50 \mu \mathrm{L}$ of sterilized distilled water $\left(\mathrm{ddH}_{2} \mathrm{O}\right)$ and stored at $-20{ }^{\circ} \mathrm{C}$. Wolbachia-infected Drosophila simulans was used as an internal control for the DNA extraction method and act as a positive control during Wolbachia screening.

We used published primers targeting approximately 438 bp of 16S rDNA Wolbachia gene (Werren \& Windsor 2000). Briefly, the PCR mixtures consisting $3 \mu \mathrm{L}$ of extracted DNA, $2.5 \mu \mathrm{L}$ of 10x PCR buffer (Invitrogen), $1 \mu \mathrm{L}$ of $\mathrm{MgCl}_{2}(50 \mathrm{mM}), 0.8 \mu \mathrm{L}$ of dNTPs $(10 \mathrm{mM}$ each), $1 \mu \mathrm{L}$ of $20 \mathrm{pmol} / \mu \mathrm{L}$ forward and reverse primers and $0.3 \mu \mathrm{L}$ of Taq DNA polymerase $(5 \mathrm{U} / \mu \mathrm{L})$. The PCR was performed on an Eppendorf Mastercycler ${ }^{\circledR}$ Pro $S$ (Eppendorf, Germany) with the following conditions: initial denaturation at $94{ }^{\circ} \mathrm{C}$ for $5 \mathrm{~min}$, followed by 30 cycles of denaturation at $94{ }^{\circ} \mathrm{C}$ for $45 \mathrm{~s}$, annealing at 57 ${ }^{\circ} \mathrm{C}$ for $45 \mathrm{~s}$, extension at $72{ }^{\circ} \mathrm{C}$ for $1 \mathrm{~min} 30 \mathrm{~s}$ and final extension at $72{ }^{\circ} \mathrm{C}$ for $10 \mathrm{~min}$. The PCR products were visualized on a $2.0 \%$ agarose gel and viewed under Gel Doc $^{\text {TM }}$ EZ System (Bio-Rad, USA). Negative controls containing $\mathrm{ddH}_{2} \mathrm{O}$ were included in every run of PCR to exclude the possibility of contamination.

\section{PCR DIGESTION}

A total reaction of $25 \mu \mathrm{L}$ containing $18 \mu \mathrm{L}$ PCR product, $2.5 \mu \mathrm{L}$ of CutSmart ${ }^{\circledR}$ buffer $(10 \mathrm{x}), 1 \mu \mathrm{L}$ restriction enzyme RsaI (New England Biolabs, USA) and water were added to the final volume. The reaction mixtures were incubated overnight or at least for $16 \mathrm{~h}$ at $37^{\circ} \mathrm{C}$. The digestion products were analyzed using $3 \%$ agarose gel electrophoresis. Restriction enzyme, $R s a \mathrm{I}$ were used to differentiate between Wolbachia supergroup A and B by cut the restriction sites $\mathrm{GT}^{\wedge} \mathrm{AC}$ which are located on 16S rDNA gene which give supergroup A with three fragments $(311,83$ and $46 \mathrm{bp})$ whereas supergroup B with five fragments $(165,146,67,46$ and 16 bp) (Pourali et al. 2009).

\section{ETHICAL APPROVAL}

Ethical approval was obtained from the Research Ethics Committee, Universiti Kebangsaan Malaysia (Project code: FF-2014-074. Reference: UKM 1.5.3.5/244/FF2014-074). 
TABLE 1. List of localities for mosquito sampling and the methods of collection used

\begin{tabular}{|c|c|c|c|c|}
\hline $\begin{array}{l}\text { Location } \\
\text { types }\end{array}$ & $\begin{array}{c}\text { District/ } \\
\text { Parliament/ State }\end{array}$ & Localities & Coordinates & Collection methods \\
\hline \multirow{12}{*}{ Urban } & \multirow[t]{2}{*}{ Batu } & Batu 5, Ipoh Road (DBKL Stor 220) & 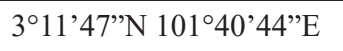 & MLTD \\
\hline & & Sentul Pasar Road (DBKL Stor 225) & 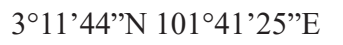 & MLTD \\
\hline & Bukit Nanas & Forested area in Bukit Nanas & $3^{\circ} 9^{\prime} 7^{\prime \prime} \mathrm{N} 101^{\circ} 42^{\prime} 17^{\prime \prime} \mathrm{E}$ & BG-Sentinel, HLC \\
\hline & Bandar Tun Razak & Velodrome Cheras (DBKL Stor 215) & 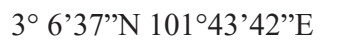 & MLTD \\
\hline & \multirow[t]{2}{*}{ Kampung Bharu } & $\begin{array}{l}\text { UKM College 4, Raja Muda Abdul } \\
\text { Aziz Road }\end{array}$ & $3^{\circ} 10^{\prime} 3$ ”N 10142’33”E & MLTD \\
\hline & & UKM College 5, Raja Abdullah Road & 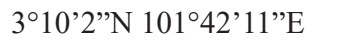 & MLTD \\
\hline & Melaka & Bendahara Road & $2^{\circ} 11^{\prime} 55^{\prime} \mathrm{N} 102^{\circ} 15^{\prime} 08^{\prime} \mathrm{E}$ & HLC \\
\hline & Seputeh & Klang Lama Road (DBKL Stor 200) & $3^{\circ} 6{ }^{\prime} 21^{\prime \prime} \mathrm{N} 101^{\circ} 40^{\prime} 40^{\prime \prime} \mathrm{E}$ & MLTD \\
\hline & Titiwangsa & UKM College 1, Temerloh Road & 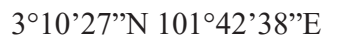 & MLTD \\
\hline & \multirow[t]{3}{*}{ Wangsa Maju } & $\begin{array}{l}\text { Genting Kelang Road (Construction } \\
\text { site) }\end{array}$ & 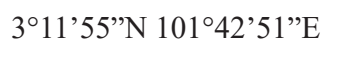 & Larva survey \\
\hline & & Seksyen 1 Block A8 & 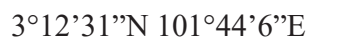 & MLTD \\
\hline & & Taman Melati Flat & 313’33”N 10143’29’'E & Larva survey \\
\hline \multirow[t]{24}{*}{ Rural } & Gombak & Ulu Gombak Forest Reserve & 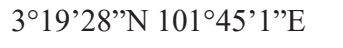 & HLC \\
\hline & Kuala Selangor & $\begin{array}{l}\text { Kilang Gula Lama Road, Tanjung } \\
\text { Karang }\end{array}$ & $3^{\circ} 24^{\prime} 47^{\prime} \mathrm{N} 101^{\circ} 9^{\prime} 55^{\prime \prime} \mathrm{E}$ & $\begin{array}{l}\text { BG-Sentinel, HLC, } \\
\text { Larva survey }\end{array}$ \\
\hline & \multirow[t]{7}{*}{ Kuantan } & Panching Road & $3^{\circ} 47^{\prime} 57^{\prime \prime} \mathrm{N} 103^{\circ} 9^{\prime} 58^{\prime \prime} \mathrm{E}$ & HLC \\
\hline & & Lembing River & & \\
\hline & & Riverview Resort & $3^{\circ} 54^{\prime} 56^{\prime \prime} \mathrm{N} 103^{\circ} 1{ }^{\prime} 54^{\prime \prime} \mathrm{E}$ & BG-Sentinel, HLC \\
\hline & & Islam Cemetery Sg. Mas & $3^{\circ} 53^{\prime} 58^{\prime \prime} \mathrm{N} 103^{\circ} 4^{\prime} 21^{\prime \prime} \mathrm{E}$ & HLC \\
\hline & & Kg. Sg. Mas (Nearby school) & $3^{\circ} 53^{\prime} 58^{\prime \prime} \mathrm{N} 103^{\circ} 4^{\prime} 34^{\prime \prime} \mathrm{E}$ & HLC \\
\hline & & Kg. Sg. Mas (Rubber Plantation) & $3^{\circ} 53^{\prime} 57^{\prime \prime} \mathrm{N} 103^{\circ} 4^{\prime} 21^{\prime \prime} \mathrm{E}$ & HLC \\
\hline & & Deer Farm & $3^{\circ} 54^{\prime} 8^{\prime \prime} \mathrm{N} 103^{\circ} 4^{\prime} 44^{\prime \prime} \mathrm{E}$ & HLC, Larva survey \\
\hline & \multirow[t]{2}{*}{ Perak } & Batu Gajah, Kinta & $4^{\circ} 27^{\prime} 34^{\prime \prime} \mathrm{N} 100^{\circ} 58^{\prime} 37^{\prime \prime} \mathrm{E}$ & HLC \\
\hline & & FELDA Gunung Besout, Sungkai & $3^{\circ} 50^{\prime} 55^{\prime \prime} \mathrm{N} 101^{\circ} 17^{\prime} 15^{\prime \prime} \mathrm{E}$ & HLC \\
\hline & Rawang & Hutan Lipur Kanching & 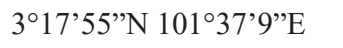 & BG- Sentinel, HLC \\
\hline & \multirow[t]{3}{*}{ Rompin } & Muadzam Shah & & \\
\hline & & Jalan Muadzam & $3^{\circ} 3^{\prime} 34^{\prime \prime} \mathrm{N} 103^{\circ} 5^{\prime} 43^{\prime \prime} \mathrm{E}$ & BG-Sentinel, HLC \\
\hline & & Desa Tanjung & $3^{\circ} 3^{\prime} 26^{\prime \prime} \mathrm{N} 103^{\circ} 5^{\prime} 24^{\prime \prime} \mathrm{E}$ & BG-Sentinel, HLC \\
\hline & Serdang & Seksyen 7, Bandar Baru Bangi & $2^{\circ} 58^{\prime} 7^{\prime} \mathrm{N} 101^{\circ} 46^{\prime} 42^{\prime \prime} \mathrm{E}$ & MLTD \\
\hline & Shah Alam & Kg. Jalan Kebun & $2^{\circ} 59^{\prime} 40^{\prime \prime} \mathrm{N} \mathrm{101³0’0’ \textrm {E }}$ & $\begin{array}{l}\text { BG-Sentinel, HLC, } \\
\text { Larva survey }\end{array}$ \\
\hline & \multirow[t]{7}{*}{ Temerloh } & Kuala Krau & & \\
\hline & & Kg. Felda Rumpun Makmur & $3^{\circ} 43^{\prime} 48^{\prime \prime} \mathrm{N} \mathrm{102} 23^{\prime} 1^{\prime \prime} \mathrm{E}$ & BG-Sentinel, HLC \\
\hline & & Kg. Lubok Wong & $3^{\circ} 46^{\prime} 2^{\prime \prime} \mathrm{N} 102^{\circ} 14^{\prime} 27^{\prime \prime} \mathrm{E}$ & HLC \\
\hline & & Kg. Paya Luas & 343’31”N 102¹9’32”'E & HLC \\
\hline & & Kg. Penderas & $3^{\circ} 43^{\prime} 49^{\prime \prime} \mathrm{N} 102^{\circ} 17^{\prime} 2^{\prime \prime} \mathrm{E}$ & HLC \\
\hline & & Kg. Terbol & $3^{\circ} 48^{\prime} 49^{\prime \prime} \mathrm{N} 102^{\circ} 13^{\prime} 45^{\prime \prime} \mathrm{E}$ & HLC \\
\hline & & Gunung Senyum (rubber plantation) & $3^{\circ} 41^{\prime} 41^{\prime \prime} \mathrm{N} 102^{\circ} 25^{\prime} 53^{\prime \prime} \mathrm{E}$ & HLC \\
\hline
\end{tabular}




\section{RESULTS}

A total of 1606 mosquitoes were collected and 765 of the mosquitoes belonging to 15 species and five genera were tested for Wolbachia detection by PCR. All samples (765 mosquitoes) were randomly chosen from the total of mosquitoes collected from each sampling sites with a minimum of 30 samples per localities. However, all samples were analysed if $n \leq 30$ for each locality. There are two types of location involved, urban (categorised into eight districts) and rural areas (nine districts) consisting of 32 localities (Table 1). As shown in Table $2,49.4 \%(378 / 765)$ of the mosquitoes were caught by HLC followed with MLTD $(34.6 \% ; 265 / 765)$, BG-Sentinel $(13.1 \% ; 100 / 765)$ and larva survey $(2.9 \% ; 22 / 765)$. BGSentinel traps showed its capability to catch a variety of species (12 species), followed with HLC (7 species), larva survey (4 species) and MLTD (2 species). In this study, Ae. albopictus has the highest number of collections which were mostly caught using HLC and MLTD methods with a total of 266 and 251, respectively. Whereas, 22 of the Ae. aegypti tested were captured during larva survey and using MLTD method.

From the 765 mosquitoes tested across all study sites, 237 (31\%) were positive for Wolbachia by PCR and more females were found to be infected with Wolbachia $(34.6 \% ; 188 / 543)$ compared to males $(22.1 \% ; 49 / 222)$. Out of 15 mosquito species tested, only five species were found to be positive for Wolbachia. The five species are Aedes albopictus, Armigeres subalbatus, Armigeres spp., Culex mimeticus and Cx. quinquefasciatus (Table 2). For Ae. albopictus, out of 600 mosquitoes tested, 189 of the mosquitoes were positive for Wolbachia with $35.8 \%$ for females (145/405) and $22.6 \%$ for males (44/195). The absence of Wolbachia in Ae. albopictus populations were shown in mosquitoes collected from Bukit Nanas $(n=19)$, Melaka $(n=2)$ and Rawang $(n=17)$. However, another species of mosquitoes collected from Bukit Nanas, Armigeres subalbatus was found to be positive for Wolbachia. Similar finding was also recorded by mosquitoes collected from Kg. Sg. Mas, Sg. Lembing (grouped into Kuantan) in which 10 out of 13 of Ar. subalbatus captured from these locations were carrying Wolbachia. Despite that, all the Ae. albopictus tested $(\mathrm{n}=10)$ from these areas was free from Wolbachia. Different findings were reported for Kg. Rumpun Makmur (grouped into Temerloh); only Ae. albopictus was positive with Wolbachia. Meanwhile, others mosquito species collected from Kg. Rumpun Makmur were all negative. The mosquito species were Aedes (Paraedes) collessi, Ar. subalbatus, Coquillettidia crassipes, Culex gelidus, Cx. hutchinsoni, Cx. mimeticus, Cx. sinensis, Mansonia annulata, Ma. indiana and Ma. uniformis. Similarly, we also found that Ae. albopictus captured from Kg. Jalan Kebun (Shah Alam) were positive with Wolbachia and a total of 11 of Ar. subalbatus collected were tested negative for Wolbachia. However, Kg. Jalan Kebun recorded a very low infection rate of $8.5 \%$ for Ae. albopictus (4/47).

TABLE 2. List of mosquitoes species captured by four different collection methods and the status of Wolbachia infection

\begin{tabular}{|c|c|c|c|c|c|c|c|c|c|c|c|c|c|}
\hline \multirow{3}{*}{ Mosquito species } & \multicolumn{4}{|c|}{ Method of of Collections } & \multirow{2}{*}{\multicolumn{2}{|c|}{$\begin{array}{c}\text { Total } \\
\text { mosquitoes } \\
\text { tested }\end{array}$}} & \multirow{3}{*}{$\begin{array}{l}\text { Status of } \\
\text { infection }\end{array}$} & \multirow{2}{*}{\multicolumn{2}{|c|}{ Wolbachia Supergroup }} & \multirow{2}{*}{\multicolumn{2}{|c|}{$\begin{array}{c}\text { Untested } \\
\text { (But positive } \\
\text { for } \\
\text { Wolbachia) * }\end{array}$}} & \multirow{2}{*}{\multicolumn{2}{|c|}{$\begin{array}{l}\text { Uninfected } \\
\text { Mosquitoes }\end{array}$}} \\
\hline & \multirow{2}{*}{$\begin{array}{c}\text { BG } \\
\text { Sentinel }\end{array}$} & \multirow{2}{*}{ HLC } & \multirow{2}{*}{$\begin{array}{c}\text { Larva } \\
\text { survey }\end{array}$} & \multirow{2}{*}{ MLTD } & & & & & & & & & \\
\hline & & & & & Male & Female & & Male & Female & Male & Female & Male & Female \\
\hline Aedes aegypti & 0 & 0 & 8 & 14 & 7 & 15 & - & - & - & - & - & 7 & 15 \\
\hline Aedes albopictus & 78 & 266 & 5 & 251 & 195 & 405 & + & 41 (A,B,AB) & $106(\mathrm{~A}, \mathrm{~B}, \mathrm{AB})$ & 3 & 39 & 151 & 260 \\
\hline $\begin{array}{l}\text { Aedes (Paraedes) } \\
\text { collessi }\end{array}$ & 0 & 1 & 0 & 0 & 0 & 1 & - & - & - & - & - & 0 & 1 \\
\hline Armigeres subalbatus & 22 & 47 & 0 & 0 & 1 & 68 & + & $1(\mathrm{~A})$ & $29(\mathrm{~A}, \mathrm{AB})$ & 0 & 6 & 0 & 33 \\
\hline Armigeres spp. & 5 & 0 & 0 & 0 & 0 & 5 & + & - & $1(\mathrm{~A})$ & 0 & 0 & 0 & 4 \\
\hline $\begin{array}{l}\text { Coquillettidia } \\
\text { crassipes }\end{array}$ & 0 & 5 & 0 & 0 & 0 & 5 & - & - & - & - & - & 0 & 5 \\
\hline Culex gelidus & 5 & 0 & 0 & 0 & 0 & 5 & - & - & - & - & - & 0 & 5 \\
\hline Culex hutchinsoni & 16 & 0 & 0 & 0 & 12 & 4 & - & - & - & - & - & 12 & 4 \\
\hline Culex mimeticus & 1 & 0 & 7 & 0 & 4 & 4 & + & $1(\mathrm{~B})$ & $1(\mathrm{~B})$ & 0 & 0 & 3 & 3 \\
\hline $\begin{array}{l}\text { Culex } \\
\text { quinquefasciatus }\end{array}$ & 8 & 1 & 2 & 0 & 3 & 8 & + & $3(\mathrm{~B})$ & $6(\mathrm{~B})$ & 0 & 0 & 0 & 2 \\
\hline Culex sinensis & 1 & 0 & 0 & 0 & 0 & 1 & - & - & - & - & - & 0 & 1 \\
\hline Culex sitiens & 1 & 14 & 0 & 0 & 0 & 15 & - & - & - & - & - & 0 & 15 \\
\hline Mansonia annulata & 2 & 0 & 0 & 0 & 0 & 2 & - & - & - & - & - & 0 & 2 \\
\hline Mansonia indiana & 1 & 0 & 0 & 0 & 0 & 1 & - & - & - & - & - & 0 & 1 \\
\hline Mansonia uniformis & 1 & 3 & 0 & 0 & 0 & 4 & - & - & - & - & - & 0 & 4 \\
\hline \multirow{3}{*}{ Total } & \multirow{3}{*}{$\begin{array}{c}100 \\
(13.1 \%)\end{array}$} & \multirow{3}{*}{$\begin{array}{c}378 \\
(49.4 \%)\end{array}$} & \multirow{3}{*}{$\begin{array}{c}22 \\
(2.9 \%)\end{array}$} & \multirow{3}{*}{$\begin{array}{c}265 \\
(34.6 \%)\end{array}$} & 222 & 543 & & 46 & 143 & 3 & 45 & $\begin{array}{c}173 \\
(78 \%)\end{array}$ & $\begin{array}{c}355 \\
(65 \%)\end{array}$ \\
\hline & & & & & \multirow{2}{*}{\multicolumn{2}{|c|}{765}} & & \multicolumn{2}{|c|}{189} & \multicolumn{2}{|c|}{48} & & \\
\hline & & & & & & & & \multicolumn{2}{|c|}{$237(31 \%)$} & & & & 528 \\
\hline
\end{tabular}

* Mosquitoes were positive for Wolbachia but supergrouping was not performed 
The prevalence of Wolbachia were focused on the Ae. albopictus populations categorised in two types of location, urban and sub-urban or rural areas (Table 3). The higher prevalence of Wolbachia were observed from Serdang with $95 \%(19 / 20)$ of the mosquitoes collected were carrying Wolbachia followed by Perak (73.3\%; 22/30), Bandar Tun Razak (69.4\%; 25/36), Wangsa Maju $(68.4 \% ; 26 / 38)$ and Muadzam Shah $(50 \%$; 20/40). Other locations showed a lower rate of infection within the range of 4 to $40 \%$. We also found that 4 out of 14 Wolbachia positive locations showed the presence of all three Wolbachia supergroups. As shown in Table 3, Bandar Tun Razak recorded almost equal number of Wolbachia infection among both males and females. Meanwhile, Ae. albopictus from Titiwangsa and Kuantan was predominantly superinfected with both $\mathrm{A}$ and $\mathrm{B}$; and followed by single infection. Meanwhile, mosquitoes collected from other three locations were only found to be superinfected with Wolbachia (AB), which were the Seputeh (2/5), Gombak (1/12) and Muadzam Shah (20/40) groups. Interestingly, only three locations showed males with supergroup A single infection of Wolbachia which were Bandar Tun Razak (3/25), Titiwangsa (3/16) and Kuantan (1/9). In addition, five locations have showed single infection of females with Wolbachia from supergroup A. Meanwhile, single infection of males with supergroup B were also found at five locations and females at seven locations. Nonetheless, more males were singly infected by supergroup B with $60.7 \%$ (17/28) infection rate as compared to females $(39.3 \% ; 11 / 28)$.

TABLE 3. Status of Wolbachia supergroup infection of Aedes albopictus in several districts in Malaysia

\begin{tabular}{|c|c|c|c|c|c|c|c|c|c|c|c|c|}
\hline \multirow{3}{*}{$\begin{array}{l}\text { Location } \\
\text { types }\end{array}$} & \multirow{3}{*}{$\begin{array}{c}\text { District/ } \\
\text { Parliament/ State }\end{array}$} & \multirow{3}{*}{$\begin{array}{c}\text { Total } \\
\text { mosquito } \\
\text { tested } \\
\text { (Positive } \\
\text { Wolbachia) }\end{array}$} & \multicolumn{6}{|c|}{ Wolbachia Supergroup } & \multicolumn{2}{|c|}{$\begin{array}{l}\text { Untested positive } \\
\text { samples }\end{array}$} & \multicolumn{2}{|c|}{$\begin{array}{l}\text { Non- infected } \\
\text { Mosquito }\end{array}$} \\
\hline & & & \multicolumn{2}{|c|}{ A } & \multicolumn{2}{|c|}{ B } & \multicolumn{2}{|c|}{$\mathrm{AB}$} & \multirow[b]{2}{*}{ Male } & \multirow[b]{2}{*}{ Female } & \multirow[b]{2}{*}{ Male } & \multirow[b]{2}{*}{ Female } \\
\hline & & & Male & Female & Male & Female & Male & Female & & & & \\
\hline \multirow[t]{8}{*}{ Urban } & Batu & $27(2)$ & 0 & 0 & 0 & 1 & 0 & 1 & 0 & 0 & 7 & 18 \\
\hline & Bukit Nanas & $19(0)$ & 0 & 0 & 0 & 0 & 0 & 0 & 0 & 0 & 1 & 18 \\
\hline & Bandar Tun Razak & $36(25)$ & 3 & 3 & 5 & 3 & 2 & 7 & 0 & 2 & 0 & 11 \\
\hline & Kampung Bharu & $53(17)$ & 0 & 0 & 1 & 1 & 1 & 14 & 0 & 0 & 27 & 9 \\
\hline & Seputeh & $5(2)$ & 0 & 0 & 0 & 0 & 0 & 2 & 0 & 0 & 2 & 1 \\
\hline & Titiwangsa & $75(16)$ & 3 & 2 & 0 & 1 & 2 & 5 & 1 & 2 & 35 & 24 \\
\hline & Wangsa Maju & $38(26)$ & 0 & 3 & 5 & 1 & 6 & 3 & 1 & 7 & 3 & 9 \\
\hline & Melaka & $2(0)$ & 0 & 0 & 0 & 0 & 0 & 0 & 0 & 0 & 0 & 2 \\
\hline \multirow[t]{9}{*}{ Rural } & Gombak & $12(1)$ & 0 & 0 & 0 & 0 & 0 & 1 & 0 & 0 & 7 & 4 \\
\hline & Kuala Selangor & $25(1)$ & 0 & 0 & 0 & 0 & 0 & 0 & 0 & 1 & 17 & 7 \\
\hline & Rawang & $17(0)$ & 0 & 0 & 0 & 0 & 0 & 0 & 0 & 0 & 4 & 13 \\
\hline & Serdang & $20(19)$ & 0 & 0 & 3 & 1 & 5 & 10 & 0 & 0 & 1 & 0 \\
\hline & Shah Alam & $47(4)$ & 0 & 0 & 0 & 0 & 0 & 3 & 0 & 1 & 15 & 28 \\
\hline & Perak & $30(22)$ & 0 & 5 & 0 & 0 & 0 & 8 & 1 & 8 & 2 & 6 \\
\hline & Kuantan & $27(9)$ & 1 & 1 & 0 & 2 & 0 & 3 & 0 & 2 & 3 & 15 \\
\hline & Rompin & $40(20)$ & 0 & 0 & 0 & 0 & 1 & 18 & 0 & 1 & 11 & 9 \\
\hline & Temerloh & $127(25)$ & 0 & 0 & 3 & 1 & 0 & 6 & 0 & 15 & 16 & 86 \\
\hline \multirow[t]{3}{*}{ Total } & & $600(189)$ & 7 & 14 & 17 & 11 & 17 & 81 & 3 & 39 & 151 & 260 \\
\hline & & & \multicolumn{2}{|c|}{$\begin{array}{c}21 \\
(11.23 \%)\end{array}$} & \multicolumn{2}{|c|}{$\begin{array}{c}28 \\
(14.97 \%)\end{array}$} & \multicolumn{2}{|c|}{$\begin{array}{c}98 \\
(52.41 \%)\end{array}$} & & & & \\
\hline & & & & & \multicolumn{2}{|c|}{147} & & & \multicolumn{2}{|c|}{42} & \multicolumn{2}{|c|}{411} \\
\hline
\end{tabular}

\footnotetext{
* Localities: Batu (DBKL MLTD Stor 220 Jalan Ipoh; DBKL MLTD Stor 225 Jalan Sentul Pasar), Bukit Nanas, Bandar Tun Razak (DBKL MLTD Stor 215 Velodrome Cheras), Kampung Bharu (UKM Residential 4 Jalan Raja Muda Abdul Aziz; UKM Residential 5 Jalan Raja Abdullah, now known as PICOMS Residential), Seputeh (DBKL MLTD Stor 200 Jalan Klang Lama), Titiwangsa (UKM Residential 1 Jalan Temerloh), Wangsa Maju (Taman Melati Apartment; DBKL houses Seksyen 1), Melaka (Jalan Bendahara), Gombak (Hutan Simpan Hulu Gombak), Kuala Selangor (Jalan Kilang Gula Lama, Tanjung Karang), Rawang (Hutan Lipur Kanching), Serdang (Seksyen 1 Bandar Baru Bangi), Shah Alam (Kg Jalan Kebun), Perak (Batu Gajah; Felda Gunung Besout), Kuantan (Sg Lembing; Sg Panching), Rompin (Muadzam Shah), Temerloh (Kg Rumpun Makmur; Kg Paya Luas; Kg Lubok Wong; Kg Penderas; Kg Terbol; Taman Eko Rimba Gunung Senyum). DBKL = Kuala Lumpur City Hall, UKM = National University of Malaysia, $\mathrm{Sg}=$ Sungai or River, $\mathrm{Kg}=$ Kampung or Village
} 
TABLE 4. Possible crossing patterns between wild mosquitoes from Bandar Tun Razak and (hypothetically) released mosquitoes containing introduced Wolbachia strain(s)

\begin{tabular}{|c|c|c|c|c|c|c|}
\hline \multirow[b]{2}{*}{ Female $(+)$} & \multicolumn{6}{|c|}{ Male $\left({ }^{\lambda}\right)$} \\
\hline & $w \mathrm{AlbA}$ & $w$ AlbB & $w \mathrm{AlbA} w \mathrm{AlbB}$ & $w \mathrm{AlbA} w \mathrm{AlbB} w \mathrm{Mel}^{\mathrm{a}}$ & $w \mathrm{Mel}^{\mathrm{b}}$ & $w R \mathrm{i}^{\mathrm{c}}$ \\
\hline$w \mathrm{AlbA}$ & $\begin{array}{l}\text { Fertile } \\
(ð 0+A)\end{array}$ & $\mathrm{Bi}-\mathrm{CI}$ & Uni-CI & $\mathrm{Bi}-\mathrm{CI}$ & $\mathrm{Bi}-\mathrm{CI}$ & $\begin{array}{c}\text { Bi-CI } \\
\text { (Incomplete } \\
\text { CI) }\end{array}$ \\
\hline$w \mathrm{AlbB}$ & $\mathrm{Bi}-\mathrm{CI}$ & $\begin{array}{l}\text { Fertile } \\
(\precsim 0+\mathrm{B})\end{array}$ & Uni-CI & $\mathrm{Bi}-\mathrm{CI}$ & & $\begin{array}{c}\text { Bi-CI } \\
\text { (Incomplete } \\
\text { CI) }\end{array}$ \\
\hline$w$ AlbAwAlbB & $\begin{array}{c}\text { Fertile } \\
(\overbrace{}^{\top}+\mathrm{AB})\end{array}$ & $\begin{array}{l}\text { Fertile } \\
(\overbrace{}^{\top}+\mathrm{AB})\end{array}$ & Fertile & $\begin{array}{c}\text { Uni-CI } \\
\text { (Complete CI) }\end{array}$ & $\begin{array}{c}\text { Bi-CI }{ }^{\mathrm{a}, \mathrm{b}} \\
\text { (Complete CI) }\end{array}$ & $\begin{array}{c}\text { Bi-CI } \\
\text { (Incomplete } \\
\text { CI) }\end{array}$ \\
\hline$w \mathrm{AlbA} w \mathrm{AlbB} w \mathrm{Mel}^{\mathrm{a}}$ & $\mathrm{Bi}-\mathrm{CI}$ & $\mathrm{Bi}-\mathrm{CI}$ & $\begin{array}{c}\text { Uni-CI } \\
\text { (Incomplete CI) }\end{array}$ & $\begin{array}{c}\text { Fertile } \\
\text { (Low hatch rate) }\end{array}$ & Fertile & - \\
\hline$w \mathrm{Mel}^{\mathrm{b}}$ & $\mathrm{Bi}-\mathrm{CI}$ & $\mathrm{Bi}-\mathrm{CI}$ & $\begin{array}{c}\text { Bi-CI } \\
\text { (Complete CI) }\end{array}$ & $\begin{array}{c}\text { Bi-CI } \\
\text { (Complete CI) }\end{array}$ & $\begin{array}{c}\text { Fertile } \\
\text { (High hatch rate) }\end{array}$ & - \\
\hline$w R \mathrm{i}^{\mathrm{c}}$ & $\begin{array}{l}\text { Bi-CI (Incomplete } \\
\text { CI) }\end{array}$ & $\begin{array}{l}\text { Bi-CI (Incomplete } \\
\text { CI) }\end{array}$ & $\begin{array}{c}\text { Bi-CI } \\
\text { (Incomplete CI) }\end{array}$ & - & - & $\begin{array}{c}\text { Fertile } \\
\text { (Half hatch } \\
\text { rate) }\end{array}$ \\
\hline Uninfected & Uni-CI & Uni-CI & $\begin{array}{c}\text { Uni-CI }^{\mathrm{b}} \\
\text { (Complete CI) }\end{array}$ & $\begin{array}{c}\text { Uni-CI } \\
\text { (Complete CI) }\end{array}$ & $\begin{array}{c}\text { Uni-CI } \\
\text { (Complete CI) }\end{array}$ & $\begin{array}{c}\text { Uni-CI } \\
\text { (Incomplete } \\
\text { CI) }\end{array}$ \\
\hline
\end{tabular}

*Supergroup AB $=$ strain $w \mathrm{AlbA}+w \mathrm{AlbB}$; Supergroup A $=$ strain $w \mathrm{AlbA}$; Supergroup B $=$ strain $w \mathrm{AlbB}$; Several pattern of crosses have been tested as annotation as (a) study by Ant and Sinkins (2018), (b) study by Blagrove et al. (2012), (c) study by Xi et al. (2006); The results of crosses were described as Complete CI which means eggs fail to hatch and Incomplete CI which means ability of eggs to hatch (but shows reduction in hatching rates)

\section{DISCUSSION}

This current paper extends from our previous article (Noor-Shazleen-Husnie et al. 2018). Here, we discussed in greater details on the types of Wolbachia infection occurred in males and females captured from different populations of mosquito. As previously reported, a lower rate of infection was recorded with $31 \%$ out of 765 mosquitoes molecularly tested were positive with Wolbachia. This study exhibited that more females (34.6\%) were infected with Wolbachia compared to males (22.1\%) (Table 2). Lower rate of infection was also reported from Thailand and Sri Lanka with positivity of $28.1 \%$ and $26.4 \%$, respectively (Kittayapong et al. 2000; Nugapola et al. 2017). Out of 13 mosquito species collected from seven provinces of Sri Lanka, only four species were detected with Wolbachia which were Ae. albopictus, Ar. subalbatus, Cx. quinquefasciatus, and Ma. uniformis. Meanwhile, a study conducted in other regions of Thailand showed a high prevalence of
Wolbachia, $61.6 \%$ ( $\mathrm{n}=1622$ tested) and 28 species out of 74 species screened were infected (Wiwatanaratanabutr et al. 2013). Several reasons can be associated with these variations of the infection rate observed. For instance, different Wolbachia detection method employed and genes selection for testing could contribute to the variability of the results. A study has shown an increase in the number of Wolbachia-positive samples after they changed into a new target DNA, $w s p$ gene from previously used, ftsZ (de Albuquerque et al. 2011). Previously, Marcon et al. (2011) have suggested that the combination of 16S rDNA and wsp targets genes is the best molecular method for Wolbachia detection that could prevent false negative results. In our study, we used 16S rDNA as the target gene and RsaI digestion to class the Wolbachia into supergroup.

Our surveillance on Ae. albopictus populations found that most of the localities were predominantly superinfected with Wolbachia by which females $(55.9 \%$; $81 / 145)$ and males $(38.6 \% ; 17 / 44)$. Both rural and urban 
areas showed the presence of all three types of Wolbachia supergroup with a high number of supergroup single infections recorded in this study. Two previous studies conducted in Malaysia reported a very low or no-single infection recorded, albeit higher rate (almost 100\%) of Wolbachia superinfection of Wolbachia were recorded from Ae. albopictus populations (Afizah et al. 2017, 2015). Although the mothers carried high density of Wolbachia, it is not confirmed that all its progeny will carry the same density of this endosymbiotic bacteria, Wolbachia. A study conducted by Ahantarig et al. (2008) showed a high-density infection of $\mathrm{F}_{0}$ mother with $w$ AlbB (supergroup $\mathrm{B}$ ) did not produce $\mathrm{F}_{1}$ (progeny) with a high-density of $w$ AlbB. The variation of Wolbachia density may plays role in the CI activation which enables the spreads of introduced strain (Wolbachia-transinfected mosquito) and Wolbachia is randomly passed through generations from mothers to male and female offspring (Ahantarig et al. 2008). However, in this study, we found a low infection rate was recorded in males as compared to females. This low infection of Wolbachia detected in males could be due to low Wolbachia density presence inside the mosquitoes making it difficult to be detected by conventional PCR method. Previously, reduction of $w A l b A$ density in males at day 5 of post-emergence has been shown, whilst the density of wAlbA infection in females were found to increase throughout maturation. Mosquitoes age, sex, and different populations play role in Wolbachia distribution (Tortosa et al. 2010).

Our study has shown a variation of Wolbachia infections detected from different localities. The variation of Wolbachia supergroups detected in populations could be due to the activation of $\mathrm{CI}$ that changed the female fitness (Sinkins 2004). Superinfected females of Ae. albopictus have the advantages of having the compatibility to mate with all types of males $(\mathrm{A}, \mathrm{B}, \mathrm{AB}$ or non-infected) and all offspring will be carrying both supergroup A and B (Dobson et al. 2004; Kittayapong et al. 2002). Kittayapong et al. (2002) showed that superinfected mother from field collection can produce progeny carrying single infection of Wolbachia either supergroup A or B (12.5\%; 10 out of 80 mothers). In our study, half of the mosquitoes collected from Rompin district were infected with both Wolbachia supergroups without the presence of single infection. This is different from Bandar Tun Razak which recorded almost equal number of Wolbachia supergroups infection in both males and females. In this condition, various possible crossmating are predicted to happen involving the activation of two types of CI which are unidirectional (Uni-CI) and bi-directional CI (Bi-CI). However, disadvantages will happen when the infected males do not harbor the same Wolbachia supergroup as in females which results in no offspring and suppresses the populations. The modification of sperm by infected Wolbachia cannot be rescued in embryo of infected females which then will activate the CI (Brelsfoard \& Dobson 2009; Dobson et al. 2004). Due to the high prevalence of superinfected Ae. albopictus in natural environment, several studies have developed the artificial Wolbachia triple-strain superinfection in Ae. albopictus. Theoretically, this will enable the activation of Uni-CI that might increase the possibility of population replacement (Ant \& Sinkins 2018; Fu et al. 2010).

We have predicted possible cross-mating that might occur in the case of Bandar Tun Razak if these artificially infected Ae. albopictus are introduced (illustrated in Table 4). All males at the Bandar Tun Razak were infected with Wolbachia. Therefore, population replacement with the introduced strain might be harder to achieve because the compatible crosses between male and female of artificially generated strains of $w \mathrm{AlbA} w \mathrm{AlbB} w \mathrm{Mel}$ will result in low number of eggs hatched (Ant \& Sinkins 2018). Meanwhile, a study by Fu et al. (2010) have successfully developed males $A e$. albopictus carrying three Wolbachia strains which are $w \mathrm{AlbA}, w \mathrm{AlbB}$ and $w \mathrm{Ri}$, and the cross-mating with natural superinfected females ( $w \mathrm{AlbA}, w \mathrm{AlbB}$ ) showed a new pattern of Uni-CI but still able to produce eggs hatching rate of $16 \%$.

Three localities of Ae. albopictus were free with Wolbachia which are mosquito collected from forested area in Bukit Nanas, Jalan Bendahara in Melaka and Hutan Lipur Kanching located at Rawang. It is difficult to conclude that Melaka group was entirely free from Wolbachia due to the low number of samples tested $(n=2)$. Therefore, a further study is needed in order to confirm this. However, Ae. albopictus from Bukit Nanas and Rawang were free of Wolbachia and the location types may play roles in the absent of Wolbachia as both localities are categorized as natural rainforest which are located at Kuala Lumpur, Capital City of Malaysia. The geographical condition become the limitation for transportation to access thus, prevent the influx of outside mosquitoes (infected) into the population that free of Wolbachia. Similar finding was also reported in a study carried out in Lahore, Pakistan which showed that out of 24 Ae. albopictus tested, none of them were positive for Wolbachia (Gulraiz et al. 2019). The study has postulated that high temperature condition during the samplings had 
caused the Wolbachia density inside mosquito to reduce which made detection difficult. Furthermore, a previous study in Panama indicated that extreme dry season had an effect towards the natural Wolbachia densities inside the beetle Chelymorpha alternans (Keller et al. 2004). In addition, the effect of constant temperatures (up to 40 ${ }^{\circ} \mathrm{C}$ ) on Wolbachia-infected eggs have been tested and reduction of Wolbachia density in adult mosquitoes was shown (Ross et al. 2019a).

Other than Ae. albopictus, Ar. subalbatus, and $C x$. quinquefasciatus, we have found that $C x$. mimeticus captured from Sg. Lembing were positive with Wolbachia from supergroup B. This study is first to report Wolbachia-positive $C x$. mimeticus, after negative infection status had been reported from previous studies (Kittayapong et al. 2000; Wiwatanaratanabutr et al. 2013). Nonetheless, Ae. aegypti collected from urban study areas showed negative infection of Wolbachia which are in line with most studies in other countries (Gulraiz et al. 2019; Kittayapong et al. 2000). Rossi et al. (2015) have postulated that the absence of Wolbachia in Ae. aegypti is associated with the presence of other types of bacteria in mosquito reproductive system known as Asaia. Symbiont bacteria, Asaia have the potential as biological control agent for vector borne diseases (Ricci et al. 2012). Previously, a Malaysian study reported the presence of Wolbachia in $25 \%$ of Ae. aegypti larvae collected from a collection site (Teo et al. 2017). Meanwhile, several studies recently have also reported the presence of natural Wolbachia from the screened Ae. aegypti (Carvajal et al. 2019; Kulkarni et al. 2019). Higher rate of infection was showed by Ae. aegypti collected from New Mexico, in which 57.4\% out of 148 was found to be infected with Wolbachia from supergroup B (Kulkarni et al. 2019). In 2019, Ross et al. (2019b) conducted cross-mating experiment involving Ae. aegypti originated from the study sites of Kulkarni et al. (2019) and detection of Wolbachia was conducted using highly sensitive molecular methods. However, the results are contrary with the findings as none of the sample was positive with Wolbachia. They postulated that crosscontamination between positive mosquitoes in previous study may contributed to the false positive results. Our study had taken several protective measures to prevent the cross contamination such as by taking extra precaution while opening the sample tubes when doing the DNA extraction. In addition, negative and positive control were always included as internal control either during PCR or DNA extraction (Noor-Shazleen-Husnie et al. 2018). Furthermore, all mosquitoes were individually tested instead of pool in group to prevent misdetection in low infected mosquito population (Kulkarni et al. 2019).

From our study, we have successfully detected Wolbachia in various species of mosquitoes such as Ae. albopictus, Ar. subalbatus, Cx. quinquefasciatus, and Cx. mimeticus by targeting $16 \mathrm{~S}$ rDNA gene. A study conducted by Wong et al. (2020) showed that most of the mosquito tested were found positive when using $16 \mathrm{~S}$ rRNA primers compared to wsp primers especially in Anopheles genera. Meanwhile, their study did not detect Wolbachia in Ar. subalbatus and only detected it in a low number of Ae. albopictus using 16S rRNA. However, our study found that Wolbachia was able to be detected using 16s rDNA primers in both of the mosquito species. Therefore, we believed that $16 \mathrm{~S}$ rDNA could be used as target gene if we would like to conduct the Wolbachia detection when involving various species of mosquitoes as first screening molecular method.

Therefore, to overcome problems of low-density detection of Wolbachia in the infected mosquito, a highly specific and sensitive molecular technique such as LAMP is required. According to Gonçalves et al. (2019), the analytical sensitivity and specificity of the LAMP assay reached $99.6 \%$ and $92.2 \%$, respectively, with a positive predictive value of $97.08 \%$ and a negative predictive value of $99.30 \%$. In fact, several studies have reported that LAMP assay can be applied as an alternative technique to replace the gold standard, PCR for Wolbachia detection when involving large-scale screening (Gonçalves et al. 2019; Noor-Shazleen-Husnie et al. 2018). We recommend this assay as a rapid, costeffective and simple method that could be applied within the field at short notice and utilised by users with limited training. All the equipment that would be required would be a hot-block or water bath (Lau et al. 2011). Reagent-wise, the costs would be similar to that of PCR, but the real advantage of this would be the rapidity of this assay, yielding results within an hour of testing, compared to 4-8 hours taken with the PCR method (Notomi et al. 2015).

\section{CONCLUSION}

Wolbachia-infected mosquito is one of the potential control approaches that would enable reduction of the use of chemical application and our reliance on insecticide. This promising approach has been used in several dengue-endemic areas in Malaysia by releasing Aedes aegypti carrying selected strain of Wolbachia. Meanwhile, Ae. albopictus must not be forgotten as they also play role in the transmission of vector-borne 
diseases especially dengue and chikungunya. In the next few years, Ae. albopictus microinjected with selected Wolbachia strain(s) might be used as vector and disease control. Therefore, baseline data on the distribution of natural Wolbachia in wild mosquito populations, including Ae. albopictus, presented in our manuscript will help to predict and provide better understanding on the outcomes of progeny when CI is activated in the wild mosquito populations. However, further studies are needed to understand the distribution of natural Wolbachia infection in Malaysia mosquito populations using molecular technique that incorporated amplification of both 16S rDNA and wsp genes with large scale of mosquito screening before the application of this biological control can be implemented widely.

\section{ACKNOWLEDGEMENTS}

We would like to thank staff from our department who were involved in the mosquito collections. Many thanks also go to Vector Unit staff from DBKL lead by Dr. Zainol Ariffin Pawanchee, for their help in providing mosquito samples collected from MLTD surveillance and larva surveys. This research was funded by UKM under Geran Galakan Penyelidik Muda (GGPM-2013-105) and Dana Fundamental PPUKM (FF-2014-074).

\section{REFERENCES}

Afizah, A.N., Vythilingam, I., Lim, Y.A., Zabari, N.Z.A.M. \& Lee, H.L. 2017. Detection of Wolbachia in Aedes albopictus and their effects on chikungunya virus. The American Journal of Tropical Medicine and Hygiene 96(1): 148-156.

Afizah, A.N., Roziah, A., Nazni, W.A. \& Lee, H.L. 2015. Detection of Wolbachia from field collected Aedes albopictus Skuse in Malaysia. Indian Journal of Medical Research 142(2): 205-210.

Ahantarig, A., Trinachartvanit, W. \& Kittayapong, P. 2008. Relative Wolbachia density of field collected Aedes albopictus mosquitoes in Thailand. Journal of Vector Ecology 33(1): 173-177.

Ant, T.H. \& Sinkins, S.P. 2018. A Wolbachia triple-strain infection generates self-incompatibility in Aedes albopictus and transmission instability in Aedes aegypti. Parasites and Vectors 11(1): 295.

Blagrove, M.S., Arias-Goeta, C., Failloux, A.B. \& Sinkins, S.P. 2012. Wolbachia strain $w \mathrm{Mel}$ induces cytoplasmic incompatibility and blocks dengue transmission in Aedes albopictus. In Proceedings of the National Academy of Sciences. pp. 255-260.

Brelsfoard, CL. \& Dobson, S.L. 2009. Wolbachia-based strategies to control insect pests and disease vectors. Asia-Pacific Journal of Molecular Biology and Biotechnology 17(3): 55-63.
Calvitti, M., Marini, F., Desiderio, A., Puggioli, A. \& Moretti, R. 2015. Wolbachia density and cytoplasmic incompatibility in Aedes albopictus: Concerns with using artificial Wolbachia infection as a vector suppression tool. PLOS ONE 10(3): e0121813.

Carvajal, T.M., Hashimoto, K., Harnandika, R.K., Amalin, D.M. \& Watanabe, K. 2019. Detection of Wolbachia in fieldcollected Aedes aegypti mosquitoes in metropolitan Manila, Philippines. Parasites and Vectors 12(1): 361.

Chen, C.D., Seleena, B., Nazni, W.A., Lee, H.L., Mohd Masri, S.M., Chiang, Y.F. \& Sofian Azirun, M. 2006. Dengue vectors surveillance in endemic areas in Kuala Lumpur city centre and Selangor state, Malaysia. Dengue Bulletin 30: 197-203.

de Albuquerque, A.L., Magalhães, T. \& Ayres, C.F.J. 2011. High prevalence and lack of diversity of Wolbachia pipientis in Aedes albopictus populations from northeast Brazil. Memórias do Instituto Oswaldo Cruz 106(6): 773-776.

Dobson, S.L., Rattanadechakul, W. \& Marsland, E.J. 2004. Fitness advantage and cytoplasmic incompatibility in Wolbachia single and superinfected Aedes albopictus. Heredity 93(2): 135-142.

Fu, Y., Gavotte, L., Mercer, D.R. \& Dobson, S.L. 2010. Artificial triple Wolbachia infection in Aedes albopictus yields a new pattern of unidirectional cytoplasmic incompatibility. Applied and Environmental Microbiology 76(17): 58875891.

Gonçalves, D., Hooker, D.J., Dong, Y., Baran, N., Kyrylos, P., Iturbe-Ormaetxe, I., Simmons, C.P. \& O’Neill, S.L. 2019. Detecting w Mel Wolbachia in field-collected Aedes aegypti mosquitoes using loop-mediated isothermal amplification (LAMP). Parasites \& Vectors 12(1): 1-5.

Gulraiz, M., Alvi, F.M., Mustafa, T., Razzaq, A. \& Latif, H.S. 2019. Distribution of Aedes aegypti, Aedes albopictus and Culex sp. and detection of Wolbachia among them in city district Lahore. Journal of Fatima Jinnah Medical University 13(2): 55-58.

Hamdan, H., Sofian-Azirun, M., Nazni, W.A. \& Lee, H.L. 2005. Insecticide resistance development in Culex quinquefasciatus (Say), Aedes aegypti (L.) and Aedes albopictus (Skuse) larvae against malathion, permethrin and temephos. Tropical Biomedicine 22: 45-52.

Hoffmann, A.A., Montgomery, B.L., Popovici, J., IturbeOrmaetxe, I., Johnson, P.H., Muzzi, F., Greenfield, M., Durkan, M., Leong, Y.S., Dong, Y. \& Cook, H. 2011. Successful establishment of Wolbachia in Aedes populations to suppress dengue transmission. Nature 476(7361): 454-457.

Keller, G.P., Windsor, D.M., Saucedo, J.M. \& Werren, J.H. 2004. Reproductive effects and geographical distributions of two Wolbachia strains infecting the Neotropical beetle, Chelymorpha alternans Boh. (Chrysomelidae, Cassidinae). Molecular Ecology 13(8): 2405-2420.

Kittayapong, P., Baisley, K., Sharpe, R., Baimai, V. \& O’Neill, S. 2002. Maternal transmission efficiency of Wolbachia superinfections in Aedes albopictus populations in Thailand. American Journal Tropical Medicine Hygiene 66(1): 103107. 
Kittayapong, P., Baisley, K.J., Baimai, V. \& O’Neill, S.L. 2000. Distribution and diversity of Wolbachia infections in Southeast Asian mosquitoes (Diptera: Culicidae). Journal of Medical Entomology 37: 340-345.

KKM 2020. Kenyataan Akhbar Ketua Pengarah Kesihatan Malaysia Mengenai Situasi Denggi, Zika dan Chikungunya di Malaysia - ME 45.2020. Malaysia: Kementerian Kesihatan Malaysia (KKM). Accessed on 22 November 2020.

KKM 2019. I-Dengue: Statistik Denggi. Malaysia: Kementerian Kesihatan Malaysia (KKM). Accessed on 22 November 2020.

Kulkarni, A., Yu, W., Jiang, J., Sanchez, C., Karna, A.K., Martinez, K.J., Hanley, K.A., Buenemann, M., Hansen, I.A., Xue, R.D. \& Ettestad, P. 2019. Wolbachia pipientis occurs in Aedes aegypti populations in New Mexico and Florida, USA. Ecology and Evolution 9(10): 6148-6156.

Lau, Y.L., Fong, M.Y., Mahmud, R., Chang, P.Y., Palaeya, V., Cheong, F.W., Chin, L.C., Anthony, C.N., Al-Mekhlafi, A.M. $\&$ Chen, Y. 2011. Specific, sensitive and rapid detection of human Plasmodium knowlesi infection by loop-mediated isothermal amplification (LAMP) in blood samples. Malaria Journal 10(1): 1-6.

Loke, S.R., Andy-Tan, W.A., Benjamin, S., Lee, H.L. \& SofianAzirun, M. 2010. Susceptibility of field-collected Aedes aegypti (L.) (Diptera: Culicidae) to Bacillus thuringiensis israelensis and temephos. Tropical Biomedicine 27: 493-450.

Luo, L., Jiang, L.Y., Xiao, X.C., Di, B., Jing, Q.L., Wang, S.Y., Tang, J.L., Wang, M., Tang, X.P. \& Yang, Z.C. 2017. The dengue preface to endemic in mainland China: The historical largest outbreak by Aedes albopictus in Guangzhou, 2014. Infectious Diseases of Poverty 6(1): 148.

Marcon, H.S., Coscrato, V.E., Selivon, D., Perondini, A.L.P. \& Marino, C.L. 2011. Variations in the sensitivity of different primers for detecting Wolbachia in Anastrepha (Diptera: Tephritidae). Brazilian Journal of Microbiology 42(2): 778-785.

Mousson, L., Zouache, K., Arias-Goeta, C., Raquin, V., Mavingui, P., Failloux, A.B. \& Lambrechts, L. 2012. The native Wolbachia symbionts limit transmission of dengue virus in Aedes albopictus. PLoS Neglected Tropical Diseases 6(12): e1989.

Nazni, W.A., Hoffmann, A.A., Noor Afizah, A., Cheong, Y.L., Mancini, M.V., Golding, N., Kamarul, G.M., Arif, M.A., Thohir, H., Nur Syamimi, H. \& Zatil Aqmar, M.Z. 2019. Establishment of Wolbachia strain $w \mathrm{AlbB}$ in Malaysian populations of Aedes aegypti for dengue control. Current Biology 29(24): 4241-4248.

Noor-Shazleen-Husnie, M.M., Emelia, O., Ahmad-Firdaus, M.S., Zainol-Ariffin, P. \& Aishah-Hani, A. 2018. Detection of Wolbachia in wild mosquito populations from selected areas in Peninsular Malaysia by loop-mediated isothermal amplification (LAMP) technique. Tropical Biomedicine 35(2): 330-346.

Notomi, T., Mori, Y., Tomita, N. \& Kanda, H. 2015. Loopmediated isothermal amplification (LAMP): principle, features, and future prospects. Journal of Microbiology 53(1): $1-5$.
Nugapola, N.N.P., De Silva, W.P.P. \& Karunaratne, S.P. 2017. Distribution and phylogeny of Wolbachia strains in wild mosquito populations in Sri Lanka. Parasites and Vectors 10(1): 230 .

Pourali, P., Roayaei, A.M., Jolodar, A. \& Razi, J.M.H. 2009. PCR screening of the Wolbachia in some arthropods and nematodes in Khuzestan province. Iranian Journal of Veterinary Research 10(3): 216-222.

Rasgon, J.L. \& Scott, T.W. 2004. An initial survey for Wolbachia (Rickettsiales: Rickettsiaceae) infections in selected California mosquitoes (Diptera: Culicidae). Journal of Medical Entomology 41(2): 255-257.

Ricci, I., Valzano, M., Ulissi, U., Epis, S., Cappelli, A. \& Favia, G. 2012. Symbiotic control of mosquito borne disease. Pathogens and Global Health 106(7): 380-385.

Ricci, I., Cancrini, G., Gabrielli, S., D'amelio, S. \& Favia, G. 2002. Searching for Wolbachia (Rickettsiales: Rickettsiaceae) in mosquitoes (Diptera: Culicidae): Large polymerase chain reaction survey and new identifications. Journal of Medical Entomology 39(4): 562-567.

Ross, P.A., Callahan, A.G., Yang, Q., Jasper, M., Arif, M.A., Afizah, A.N., Nazni, W.A. \& Hoffmann, A.A. 2019a. An elusive endosymbiont: Does Wolbachia occur naturally in Aedes aegypti? Ecology and Evolution 10(3): 1581-1591.

Ross, P.A., Ritchie, S.A., Axford, J.K. \& Hoffmann, A.A. 2019b. Loss of cytoplasmic incompatibility in Wolbachia-infected Aedes aegypti under field conditions. PLoS Neglected Tropical Diseases 13(4): e0007357.

Rossi, P., Ricci, I., Cappelli, A., Damiani, C., Ulissi, U., Mancini, M.V., Valzano, M., Capone, A., Epis, S., Crotti, E. \& Chouaia, B. 2015. Mutual exclusion of Asaia and Wolbachia in the reproductive organs of mosquito vectors. Parasites and Vectors 8(1): 278.

Rozilawati, H., Tanaselvi, K., Nazni, W.A., Masri, S.M., Zairi, J., Adanan, C.R. \& Lee, H.L. 2015. Surveillance of Aedes albopictus Skuse breeding preference in selected dengue outbreak localities, Peninsular Malaysia. Tropical Biomedicine 32(1): 49-64.

Sinkins, S.P. 2004. Wolbachia and cytoplasmic incompatibility in mosquitoes. Insect Biochemistry and Molecular Biology 34(7): 723-729.

Teo, C.H.J., Lim, P., Voon, K. \& Mak, J.W. 2017. Detection of dengue viruses and Wolbachia in Aedes aegypti and Aedes albopictus larvae from four urban localities in Kuala Lumpur, Malaysia. Tropical Biomedicine 34(3): 583-597.

Tortosa, P., Charlat, S., Labbe, P., Dehecq, J.S., Barré, H. \& Weill, M. 2010. Wolbachia age sex-specific density in Aedes albopictus: A host evolutionary response to cytoplasmic incompatibility? PLoS ONE 5(3): e9700.

Werren, J.H. \& Windsor, D.M. 2000. Wolbachia infection frequencies in insects: Evidence of a global equilibrium? In Proceedings of the Royal Society of London. Series B: Biological Sciences. pp. 1277-1285.

Wiwatanaratanabutr, I. 2013. Geographic distribution of wolbachial infections in mosquitoes from Thailand. Journal of Invertebrate Pathology 114(3): 337-340. 
WMP 2019. The World Mosquito Program's Wolbachia Method is Helping Communities Around the World Prevent the Spread of Mosquito-Borne Disease. Sri Lanka: World Mosquito Program (WMP).

Wong, M.L., Liew, J.W.K., Wong, W.K., Pramasivan, S., Hassan, N.M., Sulaiman, W.Y.W., Jeyaprakasam, N.K., Leong, C.S., Low, V.L. \& Vythilingam, I. 2020. Natural Wolbachia infection in field-collected Anopheles and other mosquito species from Malaysia. Parasites and Vectors 13(1): 1-15.

Xu, G., Dong, H., Shi, N., Liu, S., Zhou, A., Cheng, Z., Chen, G., Liu, J., Fang, T., Zhang, H. \& Gu, C. 2007. An outbreak of dengue virus serotype 1 infection in Cixi, Ningbo, People's Republic of China, 2004, associated with a traveler from Thailand and high density of Aedes albopictus. The American Journal of Tropical Medicine and Hygiene 76(6): 1182-1188.

Zainol, A.P., Ahmad, Z.Z., Norhayati, S., Umi, A., Osman, H., Awaluddin, M.A., Abdul, H., Omar, H. \& Mohd, H.M. 2009. Using mosquito larvae trapping device as an additional tool for dengue fever control in Kuala Lumpur. Malaysian Journal of Public Health Medicine 9(Suppl. 2): 34.

Zhang, D., Lees, R.S., Xi, Z., Bourtzis, K. \& Gilles, J.R.L. 2016. Combining the sterile insect technique with the incompatible insect technique: III-robust mating competitiveness of irradiated triple Wolbachia infected Aedes albopictus males under semi-field conditions. PLOS ONE 11(3): e0151864.
Zhou, W., Rousset, F. \& O'Neil, S. 1998. Phylogeny and PCRbased classification of Wolbachia strains using wsp gene sequences. Proceedings of the Royal Society of London. Series B: Biological Sciences 265: 509-515.

Noor Shazleen Husnie Mohd Mohtar, Emelia Osman \& Aishah Hani Azil*

Department of Parasitology and Medical Entomology

Faculty of Medicine

Universiti Kebangsaan Malaysia

56000 Cheras, Kuala Lumpur, Federal Territory

Malaysia

Mohd Farihan Md Yatim

Institute for Public Health

Centre for Communicable Diseases Research

National Institutes of Health

Ministry of Health

40170 Shah Alam, Selangor Darul Ehsan

Malaysia

*Corresponding author; email: aishah.azil@ppukm.ukm.edu.my

Received: 1 December 2020

Accepted: 2 May 2021 